\title{
Estrés y percepción de la capacidad de trabajar en docentes de una universidad pública
}

Stress and perception of the ability to work in teachers of a public university Estresse e percepção da capacidade de trabalhar em professores de uma universidade pública

\author{
EDWIN HERNÁN RAMÍREZ ASÍS ${ }^{1}$ \\ ROBERT PERCY JAMANCA ANAYA ${ }^{2}$
}

\begin{abstract}
RESUMEN
El objetivo de esta investigación fue identificar la relación existente entre el estrés y la capacidad de trabajo percibido en docentes universitarios, se encuestó a 217 docentes nombrados, utilizando el cuestionario de estrés ocupacional adaptado y el cuestionario de capacidad de trabajo percibida propuesta; adaptados por expertos. Se realizó la prueba de normalidad mediante Kolmogorov-Smirnov, obteniendo un $\mathrm{p}<0.05$, la cual demuestra que ambas variables no tienen distribución normal, usando el Rho de Spearman $=-0.594$ con un $\mathrm{P}$ valor de $0.00 * *$. La investigación concluye que existe una relación negativa moderada entre el estrés y la capacidad de trabajo percibida en docentes de una universidad pública.
\end{abstract}

Palabras clave: trabajo; estrés; tensión laboral; educación universitaria; ambiente de trabajo.

\begin{abstract}
The objective of this research was to identify the relationship between stress and the perceived work capacity of university teachers. 217 appointed teachers were surveyed, using the adapted occupational stress questionnaire and the work capacity questionnaire. perceived proposal; adapted by experts. The normality test was performed using Kolmogorov-Smirnov, obtaining a $\mathrm{p}<0.05$, which shows that both variables do not have a normal distribution, using Spearman's Rho $=-0.594$ with a P value of $0.00 * *$. The research concludes that there is a moderate negative relationship between stress and the perceived work capacity of teachers at a public university.
\end{abstract}

Keywords: work; stress; job strain; University education; work environment. 


\section{RESUMO}

O objetivo desta pesquisa foi identificar a relação entre estresse e a capacidade percebida de trabalho de professores universitários, foram pesquisados 217 professores nomeados, utilizando o questionário de estresse ocupacional adaptado e o questionário de capacidade de trabalho proposta percebida; adaptado por especialistas. O teste de normalidade foi realizado com Kolmogorov-Smirnov, obtendo $\mathrm{p}<0,05$, o que mostra que ambas as variáveis não apresentam distribuição normal, utilizando Rho de Spearman $=-0,594$ com valor de $p$ $0,001 * *$. A pesquisa conclui que existe uma relação negativa moderada entre estresse e a capacidade de trabalho percebida de professores de uma universidade pública.

Palavras-chave: trabalho; estresse; tensão no trabalho; Formação universitária; ambiente de trabalho.

\section{INTRODUCCIÓN}

Existe un reconocimiento internacional de que la educación es constitutiva del desarrollo económico y social de los países y que, especialmente, la educación superior representa la producción de conocimiento y aumento en ciencia y tecnología (Reis, Olivera, Santos, Guimarães, Reis, Marques \& Moura, 2017), además, el carácter estresante de la profesión del docente universitario y avalada científicamente por los expertos en el tema de la salud ocupacional (López, Del Castillo \& Oramas, 2011), por lo que, los síntomas más frecuentes son la ansiedad, la depresión y alteraciones del aparato fonológico (García \& Vélez, 2018). La idea que la carrera docente comprende riesgos asociados a la generación de trastornos psiquiátricos, es un tema de discusión en la actualidad.

Los docentes de universidades públicas peruanas se ven afectados en cuanto a la satisfacción laboral y el estado de salud, debido a los factores de estrés que conviven en su entorno laboral (El-Sayed, El-Zeiny \& Adeyemo, 2014), conocer las fuentes de estrés laboral es el primer paso que debe considerar el encargado de cualquier institución educativa (Orgambídez-Ramos, Pérez-Moreno \& Borrego-Alés, 2015) por lo tanto, basado en el análisis de 140 encuestas de docentes de 23 universidades (Păduraru, 2014) presenta las principales fuentes de estrés ocupacional que afectan en gran medida la actividad de los docentes universitario: "Salarios bajos" (la diferencia entre ingresos y carga de trabajo o entre ingresos y la calidad de la formación profesional) falta de equilibrio entre la vida laboral, personal y social (fatiga, falta de tiempo de recreación) mayor dedicación a realizar investigaciones y falta de ascenso en la carrera docente. Por otro lado, se desconoce con claridad la cantidad de docentes que sufren estrés crónico, debido a la confusión con otras patologías.

Las actividades en una universidad pública revelan que la intensificación del trabajo con mucha frecuencia interviene con la precariedad y la generación de estrés laboral (Silva, 2020), adicionalmente, la vida de un docente es un acto de equilibrio constante en el que se trata de hacer malabarismos con las responsabilidades personales y profesionales bajo el estrés generalizado de manejar las expectativas en una cultura a menudo muy competitiva (García \& Vélez, 2018), el sistema asume que los docentes deben ser lo suficientemente experimentados y resistentes como para soportar toda la presión que conlleva el trabajo, ser miembro de la facultad en una universidad puede ser una de las carreras profesionales más gratificantes, 
pero también se ha convertido en uno de los trabajos más estresantes (Lashuel, 2020). Muy pocas universidades públicas ofrecen capacitación o ayuda en la gestión de proyectos y equipos, liderazgo, tutoría y resolución de conflictos, y mucho menos en la conciencia e intervención de salud mental, en otras palabras, según Millán, Calvanese \& D’Aubeterre (2017) "aprendemos cometiendo errores que nosotros, y en cierta medida nuestros estudiantes, terminamos pagando directa o indirectamente" (p. 204), las consecuencias negativas para las universidades del estrés laboral, pueden ir de una licencia por salud temporal hasta la jubilación anticipada y una disminución constante de la calidad académica (Barbosa, Tavares \& Rodríguez, 2019). Generalmente los docentes dan muy poca importancia a la relación de trabajo y vida personal, por lo que existen algunos mayores de 50 años sin pareja ni hijos y según Barradas (2018), las exigencias académicas generan ansiedad e insomnio y esto afecta desempeño del docente universitario a nivel laboral y personal.

En el Perú, mediante la implementación de la Ley 30220 (2014) los docentes, además de la enseñanza adecuada, también deben realizar otras tareas, como realizar investigación y actividades de responsabilidad social, escribir informes y revisiones, buscar subvenciones y mejorar su visibilidad (Ley 30220, 2014). Adicionalmente, Hasan, Sameen, Gli \& Abass (2019), describen el conjunto de actividades que delimitan la vida académica del profesor: La cantidad de productos, manuales, ensayos, textos, libros, guía de prácticas, participación en eventos con presentación de trabajo, organización de eventos, formar parte de comisiones permanentes, participación como jurado evaluador y asesor de tesis, así como las disciplinas impartidas en cursos de pregrado y posgrado. En este sentido, el estrés académico se refiere al sentido de la necesidad de conocimiento y la percepción simultánea de que no hay suficiente tiempo para una vida personal, las tensiones pueden incluir la incapacidad de administrar el tiempo, la falta de habilidades digitales para adaptarse a las nuevas exigencias tecnológicas de la educación superior (Nazari \& Far, 2019; Palacios \& Montes de Oca, 2017; Fuster-Guillén, Jara-Jara, Ramírez-Asís, Maldonado-Leyva, Norabuena \& García, 2019). Los tipos de estrés según Barradas, (2018) son: agudo, agudo episódico y crónico; pueden hacernos sentir mal o incluso enfermos, pero generalmente el estrés crónico no es tomado muy seriamente. Distinguir entre estos tres tipos de estrés ayuda al docente a ver claramente sus propias circunstancias. ¿Estás exagerando, viendo una situación temporal como permanente? ¿o has estado ignorando los signos durante años? nuevamente, la culpa no ayudará, conocer los tres tipos también debería ayudarlo a encontrar una perspectiva y sentir más compasión por otras personas que están bajo estrés (Fernández, 2015).

En el contexto europeo, las autoridades públicas están prestando cada vez más atención a la promoción de la capacidad laboral a lo largo de la vida laboral y el mejor método para monitorear la capacidad laboral en poblaciones de trabajadores se está convirtiendo en una cuestión importante (El Fassi, Bocquet, Majery, Lair, Couffignal \& Mairiaux, 2013). Además, según Fernández, (2015) “Tener sobrepeso, disminuir el estado de salud, tener un trabajo muy exigente mentalmente y trabajar en una institución de gran tamaño son factores que provocan disminución en la capacidad de trabajo" (p. 105).

La capacidad de trabajo refleja las interacciones entre las características personales, las condiciones de trabajo, las capacidades funcionales de los empleados y también el estado de salud de los empleados (Mazloumi, Kazemi \& Eivazlou, 2014), la población de docentes en universidades publica está constituido por más del $50 \%$ que tiene más de 50 años 
y acompañado por las modificaciones de la Ley $\mathrm{N}^{\circ} 30220$, (2014) que dice, la edad límite para ejercer la docencia universitaria es de 75 años, por lo tanto, la edad avanzada de los docentes provocan que la capacidad de trabajo disminuya. Además, según López, Amador, González \& Álvarez (2019) el nivel de las aptitudes físicas y mentales del individuo está en función a las demandas de trabajo.

Los factores de tipo laboral como las malas condiciones ergonómicas, la mala gestión del trabajo, el bajo control sobre el trabajo y la baja satisfacción laboral se relacionan con la capacidad de trabajo no satisfactoria, diferentes autores también reconocen el efecto de las demandas psicológicas y el control sobre el trabajo en la capacidad de trabajo (Emberland y Knardahl, 2015), también, el número de enfermedades se relacionaron inversamente con la calidad del trabajo, el agotamiento emocional, el número de enfermedades explicaron el 55\% del índice de capacidad de trabajo (Redondo, 2014).

Se ha demostrado en varios estudios realizados en diferentes grupos profesionales que la edad, la obesidad, la falta de actividad física durante el tiempo libre, la baja capacidad musculoesquelética, los altos requisitos mentales, la falta de autonomía y la gran carga de trabajo físico, todos tienen un impacto negativo en el nivel de la capacidad de trabajo (El Fassi, Bocquet, Majery, Lair, Couffignal \& Mairiaux, 2013) por lo tanto, se entiende aquí por capacidad laboral según Robledo-Marín, Cardona, Segura-Cardona, Lizcano-Cardona \& Agudelo-Cifuentes (2019) al "conjunto de habilidades, destrezas, aptitudes y/o potencialidades de orden físico, mental y social, que permiten desempeñarse en un trabajo" ( $p$. 13). Para contribuir a los estudios sobre la carrera docente, buscamos responder la siguiente pregunta: ¿qué relación existe entre el estrés y la percepción de la capacidad de trabajo de los docentes en una universidad pública? El objetivo de esta investigación fue identificar la relación existente entre el estrés y la percepción de la capacidad de trabajo de los docentes de una universidad pública.

\section{METODOLOGÍA}

La investigación fue de tipo descriptivo y correlacional de enfoque cuantitativo (Hernández-Sampieri y Mendoza, 2018), se utilizó una muestra de 217 docentes nombrados de la Universidad Nacional Santiago Antúnez de Mayolo, la técnica de recolección de datos fue la encuesta y el cuestionario como instrumento, el método utilizado fue el muestreo aleatorio simple, para medir el estrés ocupacional en docentes se utilizó el Cuestionario de Estrés Ocupacional (CEO) en español adaptada por García y Vélez, (2018), dividido en tres grupos: estresores ocupacionales, tensión psicológica y recursos de afrontamiento, tiene 140 ítems, medido por una escala Likert nunca (1), a veces(2), frecuentemente(3), Casi siempre(4), Siempre(5), el cual fue validado por medio de juicio de expertos y un coeficiente de 0.879 de Alfa de Cronbach.

En cuanto a la capacidad de trabajo percibido se utilizó una adaptación del cuestionario de capacidad de trabajo percibida (CTP), la versión en castellano de este instrumento fue obtenida y validada por López, Del Castillo \& Oramas (2011), comprende un total de 10 preguntas agrupadas en 7 ítems: estimación subjetiva de la capacidad de trabajo actual comparada con la mejor de por vida ( 0 a 10 puntos), valoración subjetiva de la capacidad de trabajo en relación con las demandas físicas y mentales de trabajo (2 a 10 puntos), 
número de enfermedades diagnosticadas por el médico ( 1 a 7 puntos), estimación de las limitaciones para el trabajo por enfermedad ( 1 a 6 puntos), ausentismo por enfermedad en los últimos 12 meses ( 1 a 5 puntos), pronóstico de la capacidad de trabajo pasados dos años (1, 4, 7 puntos) y recursos mentales (disfrute cotidiano, actividad y buen ánimo, optimismo) ( 1 a 4 puntos), la puntuación total del cuestionario oscila en el rango de 7 a 49 puntos, se utilizó el punto de corte de pobre (0-14 puntos), moderada [15,29], buena [30,49].

Se cumplió con la entrega del consentimiento informado y el cuestionario fue aplicado de manera individual en el lugar de trabajo, seguidamente se ingresó a una base de datos en el programa SPSS V24, se utilizó la estadística descriptiva mediante tablas cruzadas para identificar los niveles de estrés ocupacional y la capacidad de trabajo; para la contrastar la hipótesis se realizó la prueba de normalidad de Kolmogorov-Smirnov, obteniendo un $\mathrm{p}<0.05$, la cual demuestra que ambas variables no tienen distribución normal, por lo que, para determinar la relación entre las dos variables se utilizó el estadístico Rho de Spearman.

\section{RESULTADOS Y DISCUSIÓN}

El promedio de edad fue de 57 años y la desviación estándar de 3.54; en la muestra se observa un $64 \%$ varones y un $36 \%$ mujeres; Del total de docentes un $68 \%$ eran de categoría principal y un $23 \%$ de categoría asociado y solo el $9 \%$ docentes auxiliares; Respecto a los grados el $56 \%$ tiene grado de doctor, el $14 \%$ grado de maestría y el $30 \%$ solo tiene grado de bachiller.

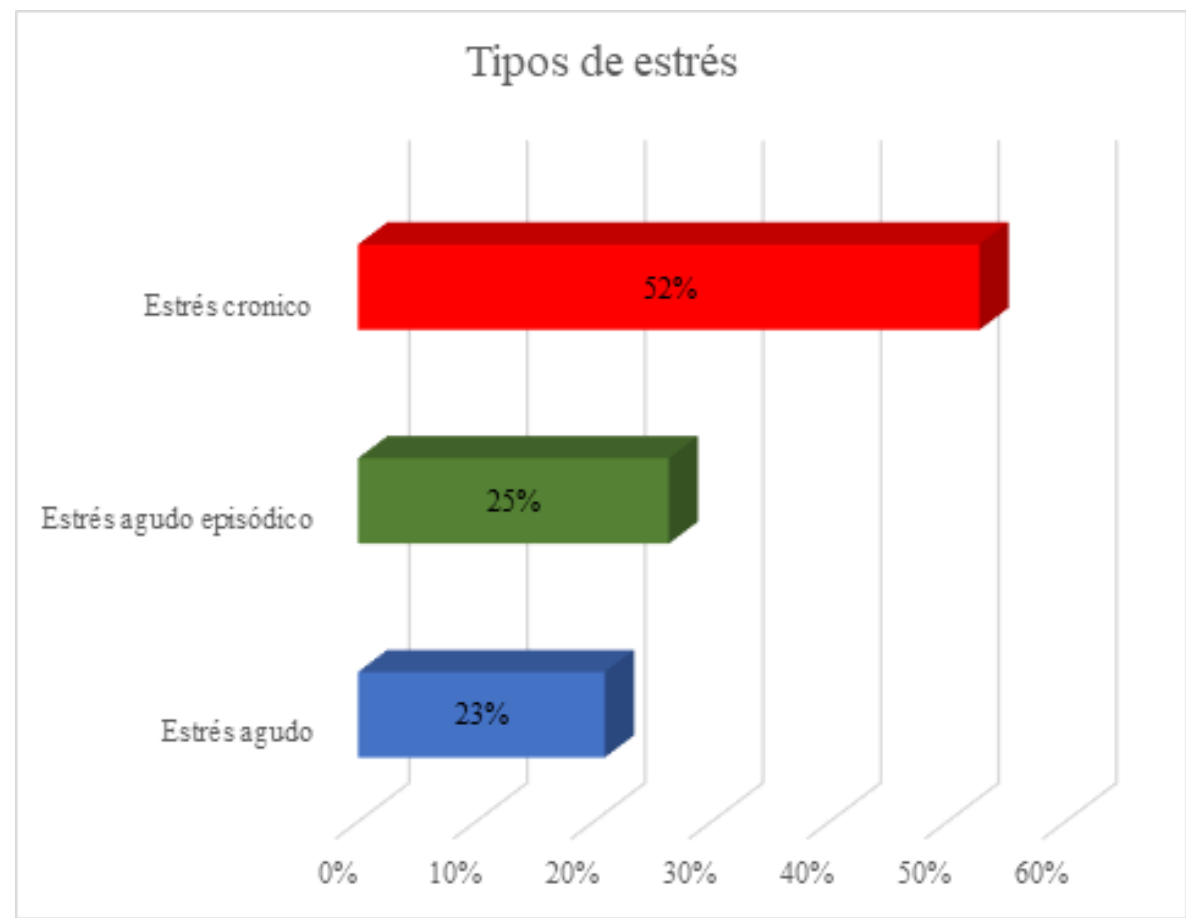

Figura 1. Tipos de estrés en docentes universitarios

Se ha identificado que el $52 \%$ de docentes universitarios presentan estrés crónico y solo el $23 \%$ percibe estrés agudo. 


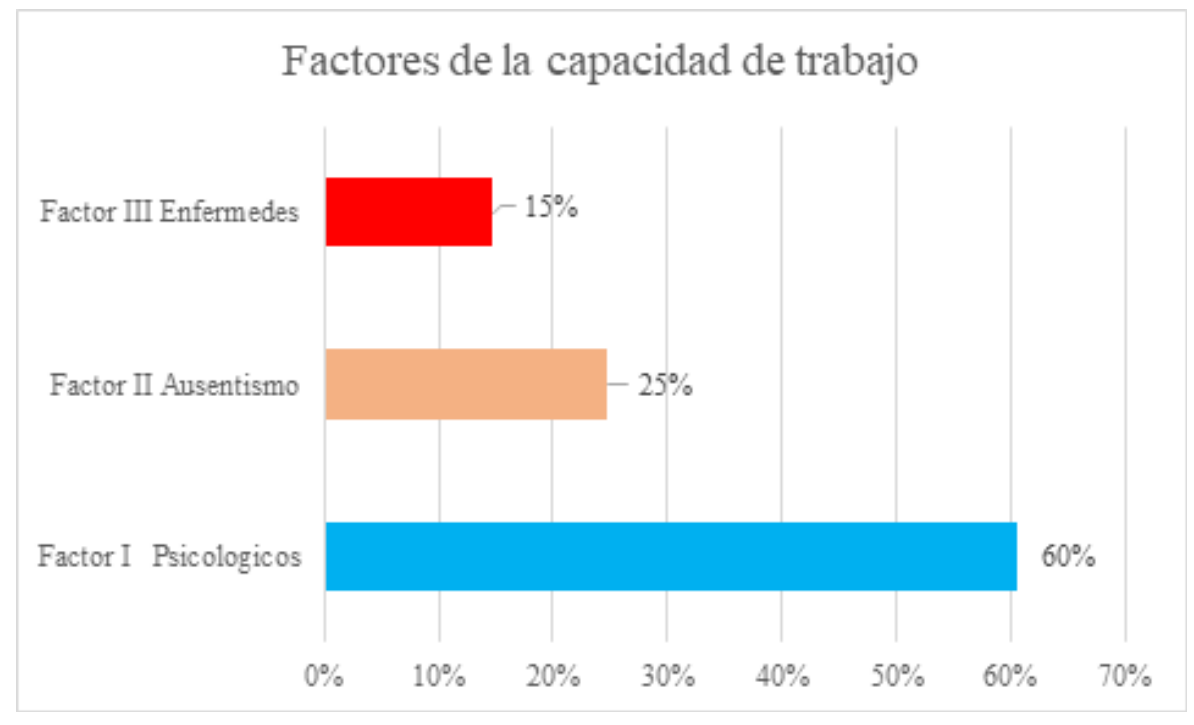

Figura 2. Percepción de los factores de la capacidad de trabajo

La capacidad de trabajo percibida presenta tres factores, el más resaltante es el factor psicológico y el menos relevante es el factor relacionado con las enfermedades.

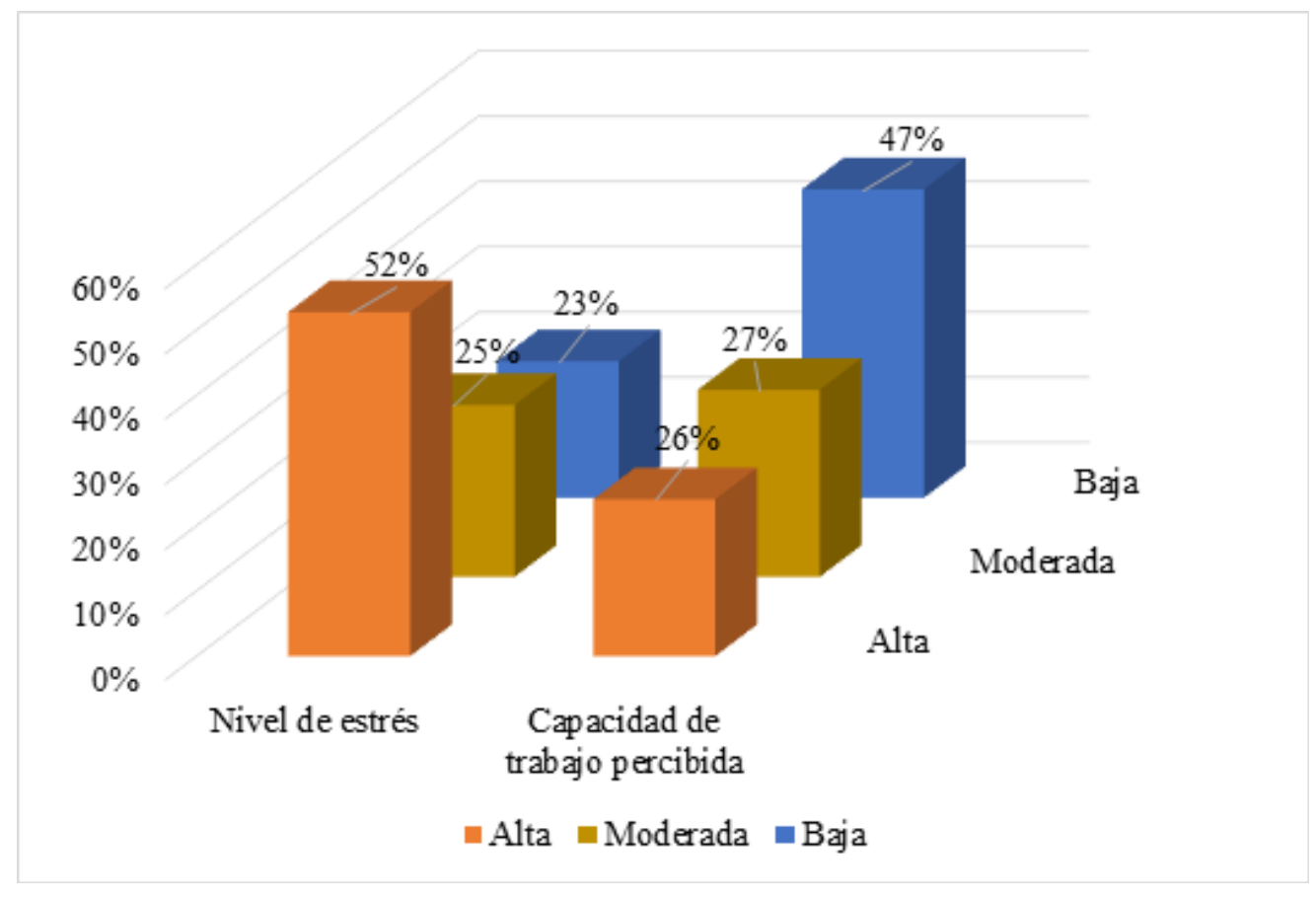

Figura 3. Capacidad de trabajo percibida según niveles de estrés

Se ha identificado que el $52 \%$ de docentes universitarios presentan estrés crónico y solo el $23 \%$ percibe estrés agudo. Además, os niveles de capacidad de en docentes universitarios el $47 \%$ percibe un nivel pobre y solo el $26 \%$ presenta un nivel de capacidad de trabajo buena. 
Tabla 1.

Capacidad de trabajo percibido según niveles de estrés

\begin{tabular}{lcccc}
\hline \multirow{2}{*}{ Estrés ocupacional } & \multicolumn{3}{c}{ Capacidad de trabajo percibida } & \multirow{2}{*}{ Total } \\
\cline { 2 - 4 } & Buena & Moderada & Pobre & \\
\hline Alta tensión & $6 \%$ & $8 \%$ & $38 \%$ & $52 \%$ \\
Activo & $7 \%$ & $15 \%$ & $3 \%$ & $25 \%$ \\
Baja tensión & $13 \%$ & $4 \%$ & $6 \%$ & $23 \%$ \\
\hline Total & $26 \%$ & $27 \%$ & $47 \%$ & $100 \%$ \\
\hline
\end{tabular}

De acuerdo a la tabla 1 se observa que los docentes que presentan un nivel alto de tensión son los que perciben una capacidad de trabajo pobre con un $38 \%$, pero existe un $13 \%$ con baja tensión y percibe una capacidad de trabajo buena.

Tabla 2.

Prueba de correlación

\begin{tabular}{|ll|r|r|}
\hline \multicolumn{1}{|c|}{ Rho de Spearman } & $\begin{array}{r}\text { Estrés Ocu- } \\
\text { pacional }\end{array}$ & $\begin{array}{r}\text { Capacidad de } \\
\text { trabajo percibida }\end{array}$ \\
\hline Estrés Ocupacional & Coeficiente de correlación & 1,000 &,$- 594^{* *}$ \\
& Sig. (bilateral) & &, 000 \\
& $\mathrm{~N}$ & 217 & 217 \\
\hline Capacidad de traba- & Coeficiente de correlación &,$- 594^{* *}$ & 1,000 \\
jo percibida & $\mathrm{N}$ &, 000 & 217 \\
\hline$* *$
\end{tabular}

**. La correlación es significativa en el nivel 0,01 ( 2 colas).

Mediante el Rho de Spearman $=-0.594$ con un $\mathrm{P}$ valor de $0.00 * *$ se demuestra la hipótesis: existe una relación negativa moderada entre el estrés y la percepción de la capacidad de trabajo percibida en docentes de una universidad pública.

Este estudio proporciona una nueva perspectiva sobre el estrés ocupacional al examinar mediante tres dimensiones y 14 indicadores en los docentes de una universidad pública, se demuestra un nivel de estrés crónico generado principalmente por la sobrecarga ocupacional, el nivel de responsabilidad, tensiones físicas y bajo nivel de recreación, estos resultados son similares a lo presentado por Barbosa, Tavares \& Rodríguez (2019), donde expone que las horas de trabajo de los docentes universitarios regulares parecen no ser suficientes, pero se ven obligadas a llevar el trabajo a casa y no tienen tiempo para el ocio, la actividad física y la vida familiar, con el consiguiente aumento de su nivel de estrés y riesgo de enfermedad. Además, complementan lo identificado por Hasan, Sameen, Gli \& Abass (2019) los resultados muestran que los profesores universitarios de Kirkuk experimentaron un nivel moderado en subescalas de agotamiento y sugiere formular políticas públicas para organizar la carrera docente teniendo en cuenta la incidencia del estrés.

Por otra lado, los estresores ocupacionales representan las condiciones laborales de los docentes universitarios son un factor que generan estrés crónico, como la ambigüedad en el 
rol y la sobrecarga ocupacional, por que acumulan muchas tareas, incluidas la enseñanza, la investigación, la divulgación y las actividades administrativas, este resultado es similar a Lagos, Retamal, Jaque \& Luengo-Martínez (2019) en un estudio con docentes de sexo femenino identifico que, a mejores condiciones de trabajo, menor es el nivel de estrés laboral presentado en esta población docente femenino, pero difiere totalmente de los identificado por (Chaudhry, 2013), utilizando el cálculo de ANOVA no revela diferencias estadísticamente significativas entre los diferentes tipos de estrés y las condiciones de trabajo, tales como contrato permanente y de visita. Adicionalmente Moeller \& Chung-Yan (2013) proponen que las universidades deben generar políticas de bienestar docente, esto debe involucrar actividades de recreación física e identificar a los docentes con riesgos psicológicos.

La capacidad de trabajo recibido por docentes de la universidad pública tiene un nivel pobre, esto explicado principalmente por las altas demanda físicas y mentales en el trabajo y por el ausentismo generado por situaciones de salud, este resultado concuerda con (Emberland \& Knardahl, 2015; Haven, Bouter, Smulders \& Tijdink, 2019; González \& Hernández, 2016) los resultados actuales respaldan que las demandas mecánicas más altas, como trabajar con los brazos levantados tienen efectos a largo plazo en el nivel de capacidad de trabajo. Además, la variedad de ocupaciones sugiere que los niveles de conflicto de roles, la primacía de los recursos humanos y el desafío positivo son predictivos la baja capacidad de trabajo actual y futura percibida (Emberland \& Knardahl, 2015; Grados \& Rúa, 2019).

Las relaciones observadas entre la capacidad de trabajo y las variables individuales generalmente corroboran las reportadas en la literatura, los resultados muestran una fuerte asociación entre el envejecimiento y la disminución de la capacidad laboral (El Fassi, Bocquet, Majery, Lair, Couffignal \& Mairiaux, 2013; Arastoo, Montazeri, Abdolalizadeh, Ghasemzadeh, Ahmadi \& Azizi, 2013) también, las enfermedades físicas y mentales ejercieron la influencia negativa más fuerte en la capacidad de trabajo percibida de las observaciones realizadas en la encuesta a docentes finlandeses (Mazloumi, Kazemi \& Eivazlou, 2014).

\section{CONCLUSIONES}

Los docentes de la universidad pública presentan una relación negativa moderada entre el estrés ocupacional y la capacidad de trabajo percibida. Esta realidad es similar a lo demostrado por Emberland \& Knardahl, (2015) "las altas demanda físicas y mentales generadas por situaciones de salud deficientes tienen efectos a largo plazo en un nivel bajo de la capacidad de trabajo.

Los docentes de la universidad pública presentan un nivel de estrés ocupacional de alta tensión, este resultado se asemeja a lo mostrado por Hasan, Sameen, Gli \& Abass (2019) que los profesores universitarios experimentaron un nivel moderado en subescalas de agotamiento y propone que debe formularse políticas públicas para organizar la carrera docente teniendo en cuenta la incidencia del estrés.

El nivel de capacidad de trabajo percibida en docentes de la universidad pública, es pobre. Este resultado es similar a lo presentado por Grados \& Rúa, (2019), donde mencionan que, la variedad de ocupaciones, los niveles de conflicto de roles y el desafío positivo son predictivos la baja capacidad de trabajo actual percibida. 


\section{REFERENCIAS}

Arastoo, A., Montazeri, A., Abdolalizadeh, M., Ghasemzadeh, R., Ahmadi, K., \& Azizi, A. (2013). Psychometric properties of Persian version of the Work Ability Index questionnaire. Payesh (Health Monitor), 12(5), 535-543.

Barbosa, M., Tavares, S., \& Rodrigues, E. (2019). Factors associated with perceived stress among professors at a federal public university. 2019, 17(1), 90. Revista Brasileira de Medicina do Trabalho, 7(1), 90-98. https://doi.org/10.5327/z1679443520190280

Barradas, M. E. (2018). Estrés y Burnout enfermedades en la vida actual. Palibrio.

Chaudhry, A. Q. (2013). Analysis of Occupational Stress of University Faculty to Improve the Quality of Their Work. IX. Journal of Quality and Technology Management, $I X(\mathrm{I}), 12-29$.

Congreso de la República. (2014, 09 de julio). Ley 30220 de 2014. Ley Universitaria. Diario Oficial El Peruano $\mathrm{N}^{\circ}$ 12914. https://www.sunedu.gob.pe/nueva-leyuniversitaria-30220-2014/

El Fassi, M., Bocquet, V., Majery, N., Lair, M. L., Couffignal, S., \& Mairiaux, P. (2013). Work ability assessment in a worker population: comparison and determinants of Work Ability Index and Work Ability score. BMC Public Health, 13(1), 305-314. https://doi.org/10.1186/1471-2458-13-305

El-Sayed, S. H., El-Zeiny, H. H., \& Adeyemo, D. A. (2014). Relationship between occupational stress, emotional intelligence, and self-efficacy among faculty members in faculty of nursing Zagazig University, Egypt. Journal of Nursing Education and Practice, 4(4), 183-194. https://doi.org/10.5430/jnep.v4n4p183

Emberland, J. S., \& Knardahl, S. (2015). Contribution of psychological, social, and mechanical work exposures to low work ability: a prospective study. Journal of occupational and environmental medicine, 57(3), 300-314. https://doi.org/10.1097/ jom.0000000000000353

Fernández, C. (2015). Fórmulas renovadas para la docencia superior. ACCI - Asociación Cultural y Científica Iberoamericana.

Fuster-Guillén, D., Jara-Jara, N., Ramírez-Asís, E., Maldonado-Leyva, H. Norabuena R.P., García, A. (2019) Desgaste ocupacional en docentes universitarios mediante el modelo factorial confirmatorio. Propósitos y representaciones 7(3). 198-230. https://doi.org/10.20511/pyr2019.v7n3.389

García, M., \& Vélez, H. (2018). Exploración de las características psicométricas del inventario de estrés ocupacional-OSI para el contexto colombiano. Psicogente, 21(39), 140-161. http://doi.org/10.17081/psico.21.39.2828

González, G., \& Hernández, O. (2016). Elementos que originan estrés en docentes universitarios del área económico administrativa. Revista reaxion, 3(1), 16-23. 
Grados, J., \& Rúa, J. (2019). Índice de capacidad de trabajo de las enfermeras que laboran en los establecimientos de salud de los distritos del Rímac-San Martín de PorresLos Olivos. [Tesis de Pregrado, Universidad Cayetano Heredia, Perú]. http:// repositorio.upch.edu.pe/bitstream/handle/upch/5561/Indice_GradosLopez_Jessica. pdf? sequence $=1 \&$ isAllowed $=\mathrm{y}$

Hasan, N. N., Sameen, F. Y., Gli, F. A., \& Abass, K. S. (2019). A Study of Job Burnout among Faculty Teacher at Kirkuk University. Indian Journal of Public Health Research \& Development, 10(10), 1851-1857. https://doi.org/10.5958/0976-5506.2019.03115.2

Haven, T. L., Bouter, L. M., Smulders, Y. M., \& Tijdink, J. K. (2019). Perceived publication pressure in Amsterdam: Survey of all disciplinary fields and academic ranks, 14(6), e0217931. https://doi.org/10.1371/journal.pone.0217931

Hernández-Sampieri, R., \& Mendoza, C. (2018). Metodología de la investigación. Las rutas cuantitativa, cualitativa y mixta. Mc Graw Hill.

Lagos, A. R., Retamal, M. S., Jaque, M. T., \& Luengo-Martínez, C. (2019). Condiciones de trabajo y estrés laboral en madres académicas universitarias. Revista Cubana de Salud y Trabajo, 20(3), 26-34.

Lashuel, H. A. (2020). Mental Health in Academia: What about faculty? eLife, 9(e54551). https://doi.org/10.7554/eLife.54551

López, G., Amador, F., G, R., González, A., \& Álvarez, S. (2019). Capacidad de trabajo percibida y su relación con factores individuales y laborales en trabajadores envejecidos del segundo nivel de atención de salud. Revista Cubana de Salud y Trabajo, 20(1), 11-19.

López, G., Del Castillo, N., \& Oramas, A. (2011). Validez y confiabilidad del Cuestionario Índice de Capacidad de Trabajo en su versión cubana. Revista Cubana de Salud y Trabajo, 12(2), 29-34.

Mazloumi, A., Kazemi, Z., \& Eivazlou, M. (2014). Validation and reliability study of farsi version of work ability index questionnaire. Journal of School of Public Health and Institute of Public Health Research, 12(1), 61-74. http://sjsph.tums.ac.ir/article-15114-en.html

Millán, A., Calvanese, N., \& D’Aubeterre, M. E. (2017). Condiciones de trabajo, estrés laboral, dependencia universitaria y bienestar psicológico en docentes universitarios. REDU. Revista de Docencia Universitaria, 15(1), 195-218. https://oi.org/10.4995/ redu.2017.6009

Moeller, C., \& Chung-Yan, G. A. (2013). Effects of social support on professors' work stress. International Journal of Educational Management, 27(3), 188-202. http:// dx.doi.org/10.1108/09513541311306431

Nazari, N., \& Far, D. M. (2019). The Relationship between Teaching Skills, Academic Emotion, Academic Stress and Mindset in University Student Academic Achievement Prediction: A PLS-SEM Approach. Journal of Intellectual Disability-Diagnosis and Treatment, 7(3), 119-133. https://doi.org/10.6000/2292-2598.2019.07.03.9 
Oramas, A., Almirall, P., \& Fernández, I. (2007). Estrés laboral y el síndrome de burnout en docentes venezolanos. Salud de los Trabajadores, 15(2), 71-87. https://www. redalyc.org/pdf/3758/375839287002.pdf

Orgambídez-Ramos, A., Pérez-Moreno, P., \& Borrego-Alés, Y. (2015). Estrés de rol y satisfacción laboral: examinando el papel mediador del engagement en el trabajo. Revista de Psicología del Trabajo y de las Organizaciones, 31(2), 69-77. http:// dx.doi.org/10.1016/j.rpto.2015.04.001

Păduraru, M. E. (2014). Sources of occupational stress among university professors-A case study for the Romanian universities. Revista de Management Comparat Internațional, 15(1), 49-56.

Palacios, M., \& Montes de Oca, V. (2017). Condiciones de trabajo y estrés en académicos universitarios. Ciencia \& trabajo, 19(58), 49-53. https://doi.org/10.4067/s071824492017000100049

Redondo, V. (2014). Discapacidad y capacidad laboral. Medicina y Seguridad del Trabajo, 60(1), 189-195.

Reis, K., Olivera, A., Santos, A., Guimarães, E., Reis, L., Marques, M., \& Moura, M. (2017). The new organization of labor at public universities: collective consequences of job instability on the health of teachers. Ciencia \& saude coletiva, 22(11), 3667-3676. https://doi.org/10.1590/1413-812320172211.01192016

Robledo-Marín, C., Cardona, D., Segura-Cardona, A., Lizcano-Cardona, D., \& AgudeloCifuentes, C. (2019). Capacidad laboral de las personas mayores de 50 años, en proceso de reintegración, adscritos a la Agencia para la Reincorporación y la Normalización. Revista de la Facultad Nacional de Salud Pública, 37(3), 15-24. https://doi.org/10.17533/udea.rfnsp.v37n3a03

Silva, E. (2020). Trabalho e subjetividade na universidade: Por uma visão global e multifacetada dos processos de sofrimento e adoecimento. Arquivos Analíticos de Politicas Educativas, 28(14), 1-30. https://doi.org/10.14507/epaa.28.4887 